\title{
Diagnostic Value of Thyroid Cytology: A Meta-analysis
}

\author{
Aiad Abdullah Abdulrazak \\ Assistant Professor of Pathology, Department of Pathology, Tikrit College of Medicine, University of Tikrit, Iraq
}

\begin{abstract}
Objective: Thyroid disorders are the most common endocrine disorders worldwide (4-7\% of population). Excision of all thyroid lesions is impractical. For rapid diagnosis, Fine needle aspiration cytology (FNAC) is simple, safe, rapid diagnostic procedure. However, indeterminate cases were reported occasionally where the patient cannot be confidently assigned to a manageable category.
\end{abstract}

Aim: We conducted a systematic review and meta-analysis to evaluate the diagnostic accuracy of FNAC in thyroid lesions and the correlation between cytological and histopathological diagnosis.

Method: A literature search of published studies was conducted using Medline/PubMed, reasearchgate, Scielo (scientific electronic library online), and Google Scholar as international databases, and IVSL (Iraqi Virtual Science Library) and IASJ (Iraqi Academic Scientific Journals) as national databases.

Results: A total of 24 studies are analyzed. The median sample size of the studies included was 100 (range, (20-23)5). The sensitivity and specificity ranged from 0.38 to 0.98 (pooled sensitivity: $0.81 ; 95 \%$ confidence interval (CI), 0.78-0.83) and from 0.47 to 1.00 (pooled specificity: 0.93; 95\% CI, 0.92-0.94), respectively. By SROC curve, the Q-value was 0.883 and the area under the curve (AUC) was 0.945 , indicating a high level of overall accuracy.

Conclusion: The recognized accuracy of FNAC in detecting thyroid cancers is applicable only to papillary carcinoma and not to other malignancies. Moreover, FNAC should be considered as a screening rather than diagnostic tool for follicular lesions. Thus, FNAC helps to orientate patient management rather than to provide final definitive diagnosis.

Keywords: Thyroid, cytology, meta-analysis, FNA, fine needle aspiration.

\section{Introduction}

Thyroid diseases are the most frequent endocrine disorders globally. In surgical practice, thyroid lesions are seen in $4-7 \%$ of population. ${ }^{(1)}$ Moreover, a survey of random patients undergoing neck ultrasonography $20 \%-76 \%$ of adult women was found to have at least one thyroid nodule. ${ }^{(2,3)}$

\section{Coressponding Author:}

\section{Aiad Abdullah Abdulrazak}

MBChB, MSc, FIBMSpath, Assistant Professor of

Pathology, Department of Pathology, Tikrit College of Medicine, University of Tikrit, Iraq

e-mail: aiadalkhalidi@gmail.com

Mobile: 00964-7710651794
The diagnostic challenge facing the clinician is to identify those lesions most at risk and to limit resection of benign conditions as much as is safely possible. Several diagnostic tests, such as radionuclide scanning, ultrasonography, and fine-needle aspiration (FNA) cytology have been used to highlight those patient requiring surgical intervention. FNAC has been used widely because it is safe, and readily doable in outpatient settings. Moreover, Fine-needle aspiration has also been shown to have similar or higher sensitivity and accuracy levels than frozen section examination. ${ }^{(4)}$

However, there is also evidence of limitations and pitfalls of FNA are those related to specimen adequacy, sampling techniques, the skill of the physician performing the aspiration, the experience of the pathologist interpreting the aspirate, and overlapping 
cytological features between benign and malignant follicular neoplasms. ${ }^{(5)}$

About one fifth of thyroid nodules with indeterminate cytology found to be malignant after surgery, therefore histopathological examination remains the standard modality of diagnosis. ${ }^{(6)}$

Aim: we conducted a systematic review and metaanalysis to evaluate the diagnostic accuracy of FNA in thyroid lesions and the correlation between cytological and histopathological diagnosis.

\section{Materials and Method}

Search Strategy: Our study was performed according to the meta-analyses guidelines of diagnostic tests accuracy studies. ${ }^{(7)}$

A literature search of published studies was conducted using Medline/PubMed, Reasearchgate, Scielo (scientific electronic library online), and Google Scholar as international databases, and IVSL (Iraqi Virtual Science Library) and IASJ (Iraqi Academic Scientific Journals) as national databases. The study conducted in May 2020.

The database search for eligible studies were made without any restriction (such as (publication date, language, or other restrictions). The search terms included "thyroid", "cytology", "FNA" and "fine needle aspiration". Thereafter, the titles were checked and duplicates were removed, then the abstracts were screened for potentially relevant studies. Full-text articles were then obtained for all potentially relevant studies. Additional references were obtained by checking the reference lists of included studies.

Inclusion and exclusion criteria: Candidate studies included in this meta-analysis must conform the following inclusion criteria: (1) studies concerning the diagnostic value of FNAc in thyroid diseases; (2) studies with cytohistopathological correlation. (3) Studies must contain sufficient information regarding true positive, true negative, false positive, false negative or any data from which this information were extractable.

The exclusion criteria were as follows: (1) duplicate articles; ( 2 case reports, editorials, abstracts, commentaries, (3) lack of essential data and (4) studies without cytohistopathological correlation.

Data Extraction: The full texts of all potentially relevant articles were evaluated by the author. Studies were included if they contained extractable data on correlation of cytological diagnosis with histopathological examination of lesions. Data from foreign-language articles (non-English) were extracted from English summary and/or tables. Data extracted from each study included: (1) name of first author; (2) year of publication; (3) number of patients; (4) absolute numbers in TP, FP, FN, and TN, or any data from which this information was derivable.

Statistical Analysis: All the analyses were conducted using the following softwares: Statsdirect version 3.2.10, Meta-Disc version 1.4, and Medcalc version 19.3

Statistical heterogeneity was assessed by Cochran's $\mathrm{Q}$ statistic (with a significance level of $\mathrm{p} \leq 0.1$ ) and I2 statistic with values of $25 \%, 50 \%$, and $75 \%$ indicating low, moderate, and high degrees of heterogeneity, respectively.

We used random effects model to minimize risk of heterogeneity across studies as diagnostic test accuracy studies are expected to be heterogeneous, making a fixed-effect model inappropriate. Publication bias was assessed using a funnel plot and Eggers test. ${ }^{(7)}$

In addition, a summary receiver operating characteristic (SROC) curve was constructed to investigate the impact of thresholds by the MosesShapiro-Littenberg method. The AUC was calculated to show the diagnosis authenticity. The closer the AUC was to 1.0 , the better the diagnosis authenticity was. ${ }^{(7)}$

\section{Results and Discussion}

Literature Search: The process of electronic database search yields 886 potential relevant articles (studies dealing with fine needle aspiration cytology (FNA) for diagnosis of thyroid diseases. An additional 34 eligible studies were identified by scanning references lists of these articles (total records identified $n=920$ ). Title and abstract screening carried out, duplicates and irrelevant studies (studies without cytohistopathological correlation, editorials, case reports) were excluded ( $\mathrm{n}=$ 858). After reviewing the full-text, we further excluded 38 studies for lacking necessary data (extractable data in the form of true positives, false positives, false negatives, and true negatives). Twenty-four articles are included in the present meta-analysis ${ }^{(\mathbf{8}-31)}$ 
Characteristic of the included Studies: The salient characteristics of included studies are outlined in Table 1.The selected studies were published from 1987 to 2019. A total of 24 studies are analyzed. Twenty one of these studies were published in English, one in Arabic and one in Korean. Included studies were from different countries, including India $(n=8)$; Iraq $(n=2)$, Bangladesh $(\mathrm{n}=2)$, and one study from each of, Egypt, Iran, Ireland, Korea, Kuwait, Nepal, Pakistan, Saudi Arabia, Syria, Turkey, UK, USA. All included studies compare cytological diagnoses with histopathological ones. Fine needle aspiration cytology (FNAc) was performed and interpreted before histopathology.

1- Role of thyroid FNA and aim of our study: Histopathology examination is the gold standard for thyroid cancer diagnosis. However, this diagnostic process is time-consuming, invasive, and it may expose patients to complications. The quest for simple, safe, diagnostic tool for the initial screening of patients with thyroid diseases deemed rational.

To our knowledge, our study is the first metaanalysis to pool the data regarding accuracy of FNA for the diagnosis of thyroid diseases and provide possible areas of improvement for future studies. Except for one meta-analysis carried out in India and include pediatric patients only. ${ }^{(32)}$

2- Diagnostic accuracy of thyroid FNAc: At the outset of literature retrieval, we found 24 studies reporting thyroid cases subjected to FNAc and published over a very large period (1987-2019) by Asian, European and American authors. The most significant finding was that the FNAc sensitivity in diagnosing thyroid lesions was noticeably high.

The overall sensitivity and specificity ranged from 0.38 to 0.98 (pooled sensitivity: $0.81 ; 95 \%$ confidence interval (CI), 0.78-0.83) and from 0.47 to 1.00 (pooled specificity: $0.93 ; 95 \%$ CI, 0.92-0.94), respectively. The diagnostic accuracy quantified by AUC was 0.945 (a diagnostic test is considered perfect if the AUC is $100 \%$, excellent if greater than $90 \%$, and good if greater than $80 \%$ ). All these results demonstrated that FNAc had a considerable potentiality in differentiating thyroid cancers from benign tumors and non-neoplastic lesions. (33)

However, the accuracy of a benign thyroid FNA result is difficult to establish because most patients with a benign result do not have surgery. The false-negative rates reported in the literature reflects only those patients who have their aspirated nodules being surgical excision, and thus the figures may be an undervalue of reality figures. Approximately $18 \%$ of patients who have an FNA are actually treated surgically. ${ }^{(34)}$

3- False-negative: The rate of false-negative results varies from 0 to $22.8 \%$ (mean $5 \%$ ). ${ }^{(\mathbf{9 , 2 6})}$ In most series, false negatives are due to inadequate specimens or improper preparation of the smear and, to a lesser extent, to the pathologist's inability to identify a malignant lesion or to the coexistence of two pathologies. Excluding these causes, the most common errors are due to the presence of a cystic neoplasm, followed by occult lesions and small tumor size. Adherence to strict criteria of specimen adequacy will greatly reduce the number of false negative diagnosis especially, if the benign nodules are followed by a repeated FNA over a period of time, and if ultrasound guidance is used. Large multinodular goiters may represent an important source of false negatives because in $10 \%$ of cases, a micropapillary carcinoma may be present. The probability of identifying this type of carcinoma by FNA without US guidance in this gland is practically zero because the aspiration punctures tend to be directed to palpable nodules. ${ }^{(35)}$

4- False-positive: On the other hand, false-positive diagnoses represent about 0 to $27 \%$ of the cases. This figure markedly decline if suspicious malignancies are ruled out, or even nullified in highly skilled hands. Almost false positive diagnoses are due to misinterpretation of hyperplastic nodules and Hürthle cell lesions as papillary or follicular carcinoma. ${ }^{(11-14)}$

The most common benign lesions giving false positive result are follicular adenoma (including the follicular adenoma with papillary hyperplasia) and hyalinizing trabecular tumor. ${ }^{(34)}$

5- Study Heterogeneity: We found significant heterogeneity among the 24 studies, so we used a random effect model.

6- Publication Bias: Publication Bias was ruled out from being the potential cause of the heterogeneity in studies. Funnel plot was performed to assess publication bias among the included studies. No publication bias was detected in this meta-analysis, thus heterogeneity cannot be attributed to publication bias. To further confirm the absence of publication bias we did Eggers test (Egger: bias $=3.427(95 \% \mathrm{CI}=-5.57$ to 12.43$) \mathrm{P}=0.4381)$. 
6- Summary receiver operator curve (SROC): SROC provides an overall measure of test accuracy. The Q-value, which is the point of intersection of the SROC curve with a diagonal line passing from the left upper corner to the right lower corner of the ROC space and corresponds to the highest common value of sensitivity and specificity for the given test. This point does not indicate the only or even the best combination of sensitivity and specificity for a particular clinical setting, but represents an overall measure of the discriminatory power of a test.

SROC curve demonstrates that the overall area under the curve of 0.945 (standard error: 0.0160). The SROC curve, shows the tradeoff between sensitivity and specificity. Our data showed that the SROC curve is positioned near the desirable upper left corner of the
SROC curve, and that the maximum joint sensitivity and specificity (ie, the Q-value) was 0.883 ; while the area under the curve (AUC) was 0.945, indicating a high level of overall accuracy.

7- Conclusion and recommendations: Because of its low cost, relative safety, and rapid sign-out time, FNAC facilitate prompt and appropriate clinical decisions and therapy. However, the recognized accuracy of FNAC in detecting thyroid cancers must be applied only to papillary carcinoma and not to other types of malignancies. Moreover, FNAC should be considered as a screening rather than diagnostic tool for follicular lesions. Thus, the aim of FNAC is to orientate patient management rather than to provide final definitive diagnosis.

Table (1) The characteristics of included studies

\begin{tabular}{|c|c|c|c|c|c|c|c|}
\hline $\begin{array}{l}\text { Study (First Author/ } \\
\text { reference number) }\end{array}$ & Country & Year & TP & FP & FN & $\mathbf{T N}$ & Total* (H/P Correlated) \\
\hline Agrawal $^{8}$ & India & 1995 & 13 & 2 & 4 & 47 & 100 \\
\hline Al-hashimi $^{9}$ & Iraq & 2014 & 54 & 3 & 18 & 4 & 79 \\
\hline Al-hureibi ${ }^{10}$ & Kuwait & 2003 & 26 & 13 & 42 & 115 & 196 \\
\hline Ali $^{11}$ & Iraq & 2016 & 13 & 5 & 1 & 81 & 100 \\
\hline Bagga ${ }^{12}$ & India & 2010 & 2 & 0 & 1 & 24 & 32 \\
\hline Al-rikabi ${ }^{13}$ & $\mathrm{KSA}$ & 1998 & 25 & 0 & 7 & 93 & 125 \\
\hline Godinho-Matos $^{14}$ & UK & 1992 & 11 & 0 & 4 & 9 & 28 \\
\hline Hajmanoochehri ${ }^{15}$ & Iran & 2015 & 60 & 12 & 3 & 26 & 101 \\
\hline Hathila $^{16}$ & India & 2015 & 7 & 2 & 1 & 50 & 60 \\
\hline Kanyakumari ${ }^{17}$ & India & 2018 & 6 & 0 & 1 & 62 & 69 \\
\hline Mundesad ${ }^{18}$ & Ireland & 2005 & 10 & 9 & 16 & 85 & 120 \\
\hline Muratli 19 & Turkey & 2014 & 54 & 17 & 8 & 31 & 126 \\
\hline Roy 20 & india & 2019 & 81 & 5 & 4 & 22 & 112 \\
\hline Salman $^{21}$ & Syria & 2018 & 5 & 1 & 1 & 38 & 45 \\
\hline Sengupta ${ }^{22}$ & India & 2011 & 18 & 0 & 2 & 158 & 178 \\
\hline Sharma ${ }^{23}$ & India & 2017 & 21 & 0 & 4 & 15 & 40 \\
\hline Sikder ${ }^{24}$ & Bangladesh & 2012 & 22 & 0 & 10 & 68 & 100 \\
\hline Sinna ${ }^{25}$ & Egypt & 2012 & 130 & 7 & 8 & 90 & 235 \\
\hline Asp ${ }^{26}$ & USA & 1987 & 18 & 10 & 0 & 9 & 37 \\
\hline Bamanikar 27 & India & 2014 & 6 & 0 & 6 & 92 & 104 \\
\hline Bhatta $^{28}$ & Nepal & 2012 & 6 & 1 & 1 & 12 & 20 \\
\hline Mahar ${ }^{29}$ & Pakistan & 2006 & 57 & 6 & 1 & 14 & 78 \\
\hline Shariar ${ }^{30}$ & Bangladish & 2017 & 5 & 0 & 2 & 63 & 70 \\
\hline $\mathrm{Ko}^{31}$ & Korea & 2008 & 86 & 0 & 31 & 71 & 188 \\
\hline
\end{tabular}

$* \mathrm{H} / \mathrm{P}$ correlated $=$ Number of cases in which cytohistopathology correlation done.

$\mathrm{TP}=$ true positive, $\mathrm{FP}=$ false positive, $\mathrm{FN}=$ false negative, $\mathrm{TN}=$ true negative. 
Ethical Clearance: Taken from the research ethics committee in Tikrit university/College of Medicine

Source of Funding: Self

Conflict of Interest: Nil

\section{References}

1. Rice CO. Incidence of nodules in the thyroid. a comparative study of symptomless thyroid glands removed at autopsy and hyperfunctioning goiters operatively removed. Arch Surg. 1932;24(3):50515.

2. Brander A, Viikinkoski P, Nickels J, Kivisaari L. Thyroid gland: US screening in a random adult population. Radiology. 1991;181(3):683-7.

3. Alolayan H, Alyahya Y, Altuaysi A, et al: Accuracy of fine needle aspiration cytology of thyroid compared to final histopathology in total thyroidectomy. IJMDC 2020;4(1):179-184

4. Amrikachi M, Ramzy I, Rubenfeld S, et al. Accuracy of fine-needle aspiration of thyroid. Arch Pathol Lab Med 2001;125: 4(84-48)8.

5. Gharib H. Fine needle aspiration of thyroid nodules: advantages, limitations, and effects. Mayo Clin Proc. 1994;69:44-49.

6. Chandio A, Shaikh Z, Chandio K, et al. Accuracy of FNAC in diagnosis of thyroid gland diseases. Nurs Palliat Care, 2018. Volume 3(1): 1-4

7. Lee J, Kim KW, Choi SH, et al. Systematic Review and Meta-Analysis of Studies Evaluating Diagnostic Test Accuracy: A Practical Review for Clinical Researchers-Part II. Statistical Methods of Meta-Analysis. Korean J Radiol. 2015;16(6):11(8811)96. doi:10.3348/kjr.2015.16.6.1188

8. Agrawal S. Diagnostic accuracy and role of fine needle aspiration cytology in management of thyroid nodules. J Surg Oncol 1995; 58: 1(68-72).

9. Al-Hashimi AM, Mohammad MJ. Fine Needle Aspiration Cytology in Thyroid Lesions: a Personal Experience. Diyala Journal of Medicine. 2014; 6(1): 81-85

10. Al-Hureibi KA, Al-Hureibi AA, Abdulmughni YA, et al. The diagnostic value of fine needle aspiration cytology in thyroid swellings in a university hospital, Yemen. Saudi Med J 2003;24:4(99-50)3.

11. Ali FH. Accuracy of Fine Needle Aspiration Cytology In The Diagnosis Of Different Thyroid
Lesions. Kerbala j pharmaceutical sci. 2016; 7(11): 81-94

12. Bagga PK, Mahajan NC. Fine needle aspiration cytology of thyroid swellings: How useful and accurate is it? Indian J Cancer. 2010; 47: 4(37-44)2.

13. Al-Rikabi AC, Al-Omran M, Cheema M, et al. Pattern of thyroid lesions and role of fine needle aspiration cytology (FNA) in the management of thyroid enlargement: a retrospective study from a teaching hospital in Riyadh. APMIS 1998;106: 10(69-10)74,

14. Godinho-Matos L, Kocjan G, Kurtz A. Contribution of fine needle aspiration cytology to diagnosis and management of thyroid disease. J Clin Pathol 1992;45:3(91-39)5

15. Hajmanoochehri F, Rabiee E. FNAC accuracy in diagnosis of thyroid neoplasms considering all diagnostic categories of the Bethesda reporting system: A single-institute experience. J Cytol 2015;32:2(38-43).

16. Hathila R, Patel S, Vaghela P, et al. Cytology findings of the thyroid lesions with the histopathology findings correlation. Int J Med Sci Public Health. 2016;5:6(42-64)6

17. Kanyakumari M, Pushpalatha K, Cytological evaluation of thyroid lesions and its correlation with histopathology in a teaching hospital. Indian J Pathol Oncol 2018;5(4):6(25-63)0

18. Mundasad B, Mcallister I, Carson J, Pyper P. Accuracy of fine needle aspiration cytology in diagnosis of thyroid swellings. Internet $\mathrm{J}$ Endocrinol. 2006; 2 (2):20-25

19. Muratli A, Erdogan N, Sevim S, et al S. Diagnostic efficacy and importance of fine-needle aspiration cytology of thyroid nodules. J Cytol 2014;31:73-8.

20. Roy PK, Bandyopadhyay S, Dubey AB, et al. A Comparative Study on Aspiration Cytology and Histopathology in Diagnosis of Thyroid Nodule and Its Correlation. Indian Journal of Otolaryngology and Head \& Neck Surgery. 2019 Oct;71(Suppl 1):9(97-10)01.

21. Salman R, Issa R, Ibrahim L. Diagnostic value of FNA in assessment of thyroid nodules Comparing cytology study with histology study. Tishreen University Journal for Research and Scientific Studies - Health Sciences Series. 2018; 40(6);71-88

22. Sengupta A, Pal R, Kar S, et al. Fine needle aspiration cytology as the primary diagnostic tool 
in thyroid enlargement. J Nat Sc Biol Med 2011; 2:113-8.

23. Sharma R, Verma N, Kaushal V, et al. Diagnostic accuracy of fine-needle aspiration cytology of thyroid gland lesions: A study of 200 cases in Himalayan belt. J Can Res Ther 2017;13:451-5.

24. Sikder AH, Rahman M, Abul Khair. Accuracy of Fine Needle Aspiration Cytology (FNAC) In The Diagnosis Of Thyroid Swellings. J. Dhaka National Med. Coll. Hos. 2012; 18 (02): (47-51)

25. Sinna EA, Ezzat N. Diagnostic accuracy of fine needle aspiration cytology in thyroid lesions. Journal of the Egyptian National Cancer Institute. 2012; 24, 63-70

26. Asp AA, Georgitis W, Waldron EJ, et al. Fine Needle Aspiration of the Thyroid Use in an Average Health Care Facility. Am J Med. 1987 83(3):4(8993).

27. Bamanikar S, Soraisham P, Jadhav S, et al. Cytohistology and clinical correlation of thyroid gland lesions: A 3 year study in a tertiary hospital. Clin Cancer Investig J 2014;3:2(08-12)

28. Bhatta S, Makaju R, Mohammad A. Role of fine needle aspiration cytology in the diagnosis of thyroid lesions. Journal of Pathology of Nepal. 2012; 2(3): 186-188
29. Mahar SA, Husain A, Islam N. Fine needle aspiration cytology of thyroid nodule: Diagnostic accuracy and pitfalls. J Ayub Med Coll Abbottabad. 2006;18(4):26-29.

30. Shariar S, Mahdia T, Kayes MT, et al. Efficacy of Fine Needle Aspiration Cytology in the Diagnosis of Thyroid Nodule. SSNI Med Col J. 2017; 2 (1):19-23

31. Koo J, Jung WH, Yang S, et al. Correlation and Accuracy Between Fine Needle Aspiration Cytology of Thyroid Lesions and Histopathologic Diagnosis -Analysis of 322 Histopathologically Confirmed Cases - Korean J Cytopathol 2008;19(2):1(44-15)1

32. Agarwal S, Jain D. Thyroid Cytology in India: Contemporary Review and Meta-analysis. J Pathol Transl Med. 2017;51(6):5(33-54)7.

33. Tie Y, Ma X, Zhu C, et al. Diagnostic accuracy of Raman spectroscopy in malignant and benign colorectal lesions: a meta-analysis. Int J Clin Exp Med 2016;9(11):205(83-20)593

34. Cibas, ES. Thyroid. In; Cibas, ES, Ducatman BS, eds. Cytology: diagnostic principles and clinical correlates. $3^{\text {rd }}$ ed.Philadelphia; Saunders Elsevier, 2009; 255-284.

35. Galera-Davidson H. Diagnostic problems in thyroid FNAs. Diagn Cytopathol 1997;17:4(22-42)8 